\title{
Chinese Family Influence on Adolescent Volunteerism Scale: Scale Construction and Some Pioneering Findings in Hong Kong ${ }^{\S}$
}

\author{
Ben M.F. Law ${ }^{1}$ and Daniel T.L. Shek ${ }^{*}, 2,3$ \\ ${ }^{I}$ Social Welfare Practice and Research Centre, The Chinese University of Hong Kong, Hong Kong \\ ${ }^{2}$ Centre for Quality of Life, Hong Kong Institute of Asia-Pacific Studies, Chinese University of Hong Kong, Hong Kong \\ ${ }^{3}$ Kiang Wu Nursing College of Macau, Macau
}

\begin{abstract}
The development of an instrument that measures family influence to adolescent volunteerism is reported. The Chinese Family Influence on Adolescent Volunteerism Scale (C-FIAV) was constructed. Content validity, cultural relevance, and reading level of the measure were reviewed by adolescents and a panel of experts. The scale was then administered to a sample of 5,946 junior and senior high school students. Exploratory factor analyses revealed a 2-factor structure, namely positive family influence and extrinsic family influence. Reliability analyses showed that the related measures were internally consistent. The sub-scale scores for volunteers and non-volunteers were different, indicating criterionrelated validity. The score was also associated with another measurement on self-ranked importance of family, indicating construct validity. Generally speaking, grade effect was more significant than gender effect for the C-FIAV and sub-scale scores. Implications and limitations are discussed.
\end{abstract}

Keywords: Adolescent volunteerism, family influence, Chinese, validation, measurement.

\section{INTRODUCTION}

This study aims to develop a scale named the Chinese Family Influence on Adolescent Volunteerism Scale (CFIAV). This paper reports a validation study in which the content validity, reliability, criterion-related validity, construct validity, and factor structure of this instrument were examined based on the responses of Chinese adolescents in Hong Kong. There are several sections in this paper. First, the importance of understanding family influence on adolescent volunteering behavior is highlighted. Second, the problems of existing empirical findings related to family influence are reviewed. Third, the underlying conceptual model in understanding family influence is discussed. Fourth, the psychometric properties of the C-FIAV are reviewed. Fifth, some preliminary findings of the normative findings associated with the scale, particularly the grade and gender effects, are explored.

As adolescents spend more time with peers and less with family [1], we may have prima facie skepticism towards family influence on adolescent prosocial behavior. However, most adolescents' fundamental values remain close to their parents [2]. A survey of the literature shows that two main family factors related to adolescent volunteerism were frequently researched. The first factor is family modeling. In Germany, Canada, and the United States, there was a higher chance that adolescents would volunteer if their parents volunteered [3-5]. Distinguished adolescent volunteers reported

*Address correspondence to this author at the Department of Social Work, The Chinese University of Hong Kong, Shatin, Hong Kong;

E-mail: danielshek@cuhk.edu.hk

${ }^{\S}$ The paper is part of the Ph.D. thesis of the first author under the supervision of the second author. the importance of family modeling [6]. Children with parental modeling exhibited more prosocial and volunteering behavior [7]. Parental modeling was even argued to be the strongest predictor for adolescent volunteerism [8]. Unfortunately, no scientific study has been conducted on this topic in the Chinese context. Moreover, there is no standardized validated instrument to measure family modeling effect on adolescent volunteerism.

The second area is family support. Family support includes verbal endorsement and non-verbal cues that the adolescents have the impression that family permits their participation. Parental support in terms of verbal encouragement could predict adolescent volunteering behavior in a longitudinal study [9]. In Hong Kong, families were one important source of support for adolescent volunteering [10]. In general, Hong Kong families seemed to support their adolescent children's volunteering behavior [11]. The reasons for support were allowing children to care for other people, to contribute to the society and to gain social experience [11]. Chinese adolescents should gain the parental approval or support for the participation as a manifestation of obedience. On the other hand, Chinese parents do not volunteer frequently [12]. As family modeling seldom exists, family influence in terms of support to adolescents seems to be critical. Unfortunately, there is no validated scale to measure family support on adolescent volunteerism.

When we examine the existing literature, there are substantial grounds to construct a scale to measure family influence. First, with the exception of studies on family modeling and family support, there is no attempt in differentiating other kinds of family influence. Second, there is no attempt in soliciting an exhaustive list of family influence and thus we cannot compare the impact of various kinds of family influence simultaneously. A standardized instrument desig- 
nating to measure different kinds of family influence is indispensable.

Third, it is especially important to assess family influence on adolescent volunteerism in the Chinese culture. There are two contrasting cultural forces regarding family influence on volunteerism. As far as the push factor is concerned, one important cultural system is Confucianism. According to Confucianism, all people are socially embedded. Chinese people have to concern about the social networks and have to overcome ourselves by suppressing our desires for the collective harmony of the society [13]. The highest social ideal of a man in Confucianism is ren, or compassion, humility, or benevolence. In this sense, Chinese cultural system encourages volunteering.

There are pull cultural factors. Families are central social institutions that members of a Chinese family have to satisfy the family needs before accommodating the out-groups [14]. Volunteering involves helping other people outside the family networks. Thus volunteering is not of primal importance. Another hindering factor is the importance of educational excellence. Chinese parents are well concerned about adolescent children's academic performance that other nonacademic activities are not fully encouraged, including volunteering. The resulting influence combining the push and pull factor is not known. We have to examine family influence systematically so that we can understand how Chinese adolescents' prosocial behavior is influenced by their families.

Based on an extensive review of the literature on adolescent development [12] and the first author's substantial experience working with Chinese adolescents and their families, nine kinds of family influence were designed. These include:

1. Adolescent's perception of family members' involvement in volunteer service

2. Adolescent's perception of family members' beliefs on volunteerism

3. Family support for adolescent volunteering

4. Family modeling for adolescent volunteering

5. Tangible reward to adolescent volunteering

6. Conforming to family pressure to perform volunteer service

7. Family tutorage (in terms of skill and knowledge indoctrination) to adolescent volunteering

8. Family invitation to volunteer

9. Family sponsorship to adolescent volunteering

All statements are adolescents' perception of family influence. The first two items emphasize adolescents' perception of family members' belief and behavior. The rest are adolescents' personal assessment of family influence towards their volunteering participation.

Conceptually speaking, these nine kinds of influence can be grouped into two domains. The knowledge of family members' involvement in volunteer service is a prerequisite of modeling whereas family members' beliefs on volunteerism can be transmitted to adolescents [15]. Family support in terms of words [4] and non-verbal gestures such as facial clues are positive reinforcers toward adolescents' further participation. Adolescents will participate in volunteer service owing to modeling effect. When a family member is interested in adolescent children's volunteer service, there is a chance that he or she will teach adolescent children how to serve. A notable example is to teach adolescents how to make gifts to the needy. However, family tutorage is never researched in volunteering literature. If asked, there is a higher chance for adolescents to volunteer [16]. Family invitation is also very likely. This domain of family influence is called positive family influence.

The second domain exerts different influence. According to cognitive motivational approach [17], tangible reward is not conducive to the further participation of volunteer services. Reward downgrades the meaning of services especially if the reward is more attractive than the service itself [17]. If the reward is not attractive enough there is no use to use the reward. Besides, some families may urge adolescents to volunteer to the extent that adolescents feel the pressure. They may give in and perform out of family coercions. This phenomenon is renowned among Chinese family members as a face-giving strategy [14]. Coercion, however, is a kind of deterrent of further volunteering [18]. Lastly, money is needed for adolescents to participate in volunteer service as it involves additional expense. Family sponsorship appeared to be related to young adolescent volunteering in Hong Kong [11]. However, if families use sponsorship as an inducement to adolescents' participation, it is like tangible reward that adolescents are more difficult to obtain intrinsic satisfaction from the services themselves. This domain of family influence highlights external rewards and psychological pressure as extrinsic influence.

A survey of the literature shows that there is no existing scale to measure and compare different kinds of family influence. A valid and reliable instrument is needed in research, systemic assessment and family studies. The development and validation of the C-FIAV is an essential initial step to set the stage for research on understanding family influence to adolescent volunteerism. As Bronfenbrenner's ecological perspective would suggest [19], volunteering behavior seems to be influenced by multi-leveled, interacting factors [20]. Family intervention can be derived to enhance adolescent's participation as a kind of systemic influence.

Family influence is expected to be different for different categories of adolescents. The underlying assumption is that family influence on adolescent's social behavior exists and those who are closer to the families would be affected to a greater extent. On the other hand, active volunteers' perception of family influence may also be different from nonvolunteers' perspective. First, it is hypothesized that the perception of positive family influence on volunteerism for adolescent volunteers would be stronger than that for nonvolunteers. On the other hand, the perception of extrinsic family influence should be lower for volunteers as they have experienced the intrinsic satisfaction of services. Nonvolunteers do not recognize the intrinsic satisfaction of volunteer service and would perceive volunteering as a means to attain extrinsic family rewards. Vice-versa, non-volunteers' families may attempt to use more reward and pressure to induce them to perform, with ineffective results. Second, girls are more home-oriented [21] and the influence from 
families is stronger than boys [22]. Logically speaking, it may be hypothesized that girls' perceived positive family influence to volunteerism is stronger than boys. Third, as adolescents from junior grades are closer to families than those from senior grades [2], it is hypothesized that grade difference is also prominent in C-FIAV scores: juniors' family influence is stronger than seniors.

This study attempted to develop and validate the C-FIAV for use with Chinese adolescents. The C-FIAV was initially constructed from the nine kinds of family influence mentioned. Content validity and reading level were assessed by a group of high school students and an expert panel. The factor structure of the instrument, its reliability status, and validity status (criterion-related validity and construct validity) were then examined. The criterion-related validity was conducted by analyzing the differences in C-FIAV profile scores with and without volunteering experience. The construct validity was conducted by analyzing the association between CFIAV and another personal assessment of the importance of social system influence. Finally, some preliminary score findings with gender and grade differences were reported.

\section{METHOD}

\section{Participants and Procedure}

The current study was conducted in Hong Kong Special Administrative Region (HKSAR), which is a major international city south of province of Guangdong in People's Republic of China. Five thousand nine hundred and forty-six secondary school students $(2,193$ boys $(36.9 \%)$ and 3,744 girls (63.1\%) and 9 respondents not indicating their gender) participated in the study. They were recruited from thirty-one secondary schools and one Protestant church by convenience sampling. More girls' schools were involved than boys' schools, thus explaining the gender discrepancy. Among respondents, $66 \%$ were juniors (S. 1 (Grade 7) to S. 3 (Grade 9), age range from 11 to 14 ) whereas $34 \%$ were seniors (S.4 (Grade 10) to S.6 (Grade 12), age range from 15 to 19). The mean age of respondents were 14.77 years $(\mathrm{SD}=1.60)$. As the sample comes from convenience sampling, caution must be taken to interpret the findings.

Both parental and participant consents were obtained. Parent consent was sought by sending letters to parents by the researcher upon the request of school authority. For participant consent, the respondents were asked to indicate their wish if they did not want to participate in the study at the time of administration of data collection (i.e. "passive" informed consent). All respondents completed the scales and demographic characteristics in a self-administration format with adequate time provided.

\section{Instruments}

Chinese Family Influence to Adolescent Volunteerism Scale $(C-F I A V)$. The 9-item C-FIAV is a self-reported scale. The items correspond directly to the nine kinds of family influence. It assesses the influence by self-report by using a 6-point Likert-type scale ranging from 1 (strongly disagree) to 6 (strongly agree) (Table 1 for item descriptions).

The 9-item C-FIAV was constructed by the first author. It was initially assessed by a group of potential respondents and then an expert panel with five members focusing on its content validity and cultural relevance. The primary objective of initial informal assessment by potential respondents is to assess the difficulty level of the instrument. Ten high school students (Grade 7 to 12) were recruited. In general, they agreed that the content of the scale was understandable. Yet junior graders tended to perceive some items were quite similar, such as "My family members volunteer" and "I volunteer because of my family modeling". The researcher took note of the comments and brought them to discussion in the expert panel.

The members of the expert panel included two teachers of Chinese Culture, two social workers and one high school vice-principal. The panel members fulfilled at least one of the following criteria: (1) extensive working experience in organizing services with adolescent volunteers, (2) substantial cultural knowledge, and (3) expertise in understanding

Table 1. Rotated Factor Matrix of Family Influence on Adolescent Volunteerism Scale (C-FIAV)

\begin{tabular}{|c|c|c|c|c|c|c|c|c|c|c|}
\hline \multirow{3}{*}{$\begin{array}{c}\text { Item } \\
\text { 1. My family participates in volunteering actively }\end{array}$} & \multicolumn{10}{|c|}{ Factors } \\
\hline & \multirow{2}{*}{$\begin{array}{c}\begin{array}{c}\text { One-Factor } \\
\text { Model }\end{array} \\
\underline{.49}\end{array}$} & \multicolumn{2}{|c|}{$\begin{array}{l}\text { Two-Factor } \\
\text { Model }\end{array}$} & \multicolumn{3}{|c|}{$\begin{array}{c}\text { Three-Factor } \\
\text { Model }\end{array}$} & \multicolumn{2}{|c|}{$\begin{array}{l}\text { Two-Factor Model } \\
\text { (Case } 1 \text { to 2973) }\end{array}$} & \multicolumn{2}{|c|}{$\begin{array}{l}\text { Two-Factor Model } \\
\text { (Case } 2974 \text { to 5946) }\end{array}$} \\
\hline & & .76 & .15 & .75 & .26 & .16 & .77 & .14 & .75 & .16 \\
\hline 2. My family considers volunteering meaningful & .34 & .80 & -.12 & .88 & .08 & -.03 & .80 & -.10 & .80 & -.14 \\
\hline 3. I volunteer because of my family support & .47 & .78 & .09 & .70 & .37 & .04 & .78 & .10 & .78 & .07 \\
\hline 4. I volunteer because of my family modeling & .62 & .73 & .33 & .57 & .53 & .21 & .74 & .32 & .73 & .34 \\
\hline 5. I volunteer because of material reward by family & .35 & .14 & .82 & .15 & .19 & .85 & .15 & .81 & .13 & .83 \\
\hline 6. I volunteer because of family coercion & .21 & -.03 & .82 & -.01 & .12 & .85 & -.04 & .81 & -.02 & .82 \\
\hline 7. I volunteer because of family tutorage & .64 & .65 & .47 & .31 & .81 & .20 & .65 & .46 & .64 & .49 \\
\hline 8. I volunteer because of family invitation & .65 & .64 & .49 & .30 & .81 & .21 & .64 & .47 & .64 & .50 \\
\hline 9. I volunteer because of family sponsorship & $\underline{.40}$ & .27 & .72 & .07 & .52 & .57 & .30 & .70 & .24 & .74 \\
\hline Factor & 1 & 1 & 2 & 1 & 2 & 3 & 1 & 2 & 1 & 2 \\
\hline Variance explained & $46.4 \%$ & \multicolumn{2}{|c|}{$63.97 \%$} & \multicolumn{3}{|c|}{$71.22 \%$} & \multicolumn{2}{|c|}{$63.50 \%$} & \multicolumn{2}{|c|}{$64.47 \%$} \\
\hline
\end{tabular}

Note: The highest loading among the factors for an item is underlined. 
the literacy level of high school students.

In the review of content validity, experts were required to examine the relevance of the test items, the representativeness of the scope covered by the test items and the cultural relevance. A panel meeting was conducted to examine the content validity. Prior to the panel meeting, each member received the C-FIAV and the discussion guideline. They were asked to go through the items and elicit additional thoughts of the scale. During the panel meeting, panel members raised their opinions and the decisions were based on social consensual method (consensual validity), in which the agreement within the group provides evidence for the validity of the scale.

The panel members agreed on the importance of the nine statements in assessing the family influence. The juniors' concern of differentiating observation of family members' behavior and modeling were raised. Both teachers opined that adolescents with normal range of cognitive abilities could differentiate these two aspects without great difficulties. Thus two items were retained. The discussion then shifted to two areas, the expansion of each kind of family influence and the referent of the family system. First, one area of family influence is represented by one statement. Additional items would enhance the reliability and validity. Yet adolescents would lose patience if they have to fill in similar items. Panel members agreed that no additional item was needed because it would cause duplication. Second, one expert raised the need to specify the family members as fathers, mothers, siblings and other close family members. Other experts opined that as the scale was in its initial stage of addressing family influence, family influence en masse was adequate. Once the influence was found, the differential impact with various family members could be examined. The scale retained the use of the term of "family members".

On the whole, the experts were satisfied with the relevance and representativeness of the C-FIAV items. The content of the scale fitted the characteristics of Chinese families.

Ranking of family, school and peers in influence of volunteering participation. Respondents were requested to rank the influence of family, school, and peers on the (1) decision to volunteer, and (2) the decision to continue. The most important system was ranked "1" whereas that with the least importance was ranked "3".

Volunteering experience and demographic characteristics. Respondents were requested to indicate whether they had performed any volunteer service within the twelve months. They also had to indicate their gender and their grades (junior: Grade 7 to 9; senior: Grade 10 to 12).

\section{RESULTS}

\section{Factor Structure}

Conceptually speaking, the items of C-FIAV can be categorized in terms of "positive family influence" and "extrinsic family influence" dimensions. Factor analysis is used to review whether the conceptual framework of C-FIAV is aligned with empirical findings. Exploratory factor analysis was chosen instead of confirmatory factor analysis based on several rationales. First, a detailed model relating the latent variables to the observed variables cannot be specified in advance because theoretical accounts related to family influ- ence are still not yet established. Second, the number of latent variables could not be determined in advance before the analysis. The number of variables was driven by empirical data. Third, analysis of covariance should be carried out only when basic nature of latent variables and the corresponding theories are resolved.

Principal components analysis with varimax rotation was employed. The two-factor model emerged. This explained $63.97 \%$ of variance and all eigenvalues were greater than unity. Items with highest factor loading were grouped. The result is shown in Table $\mathbf{1}$.

The first factor is the "positive family influence" including family volunteering behavior, beliefs, support, modeling, tutorage and invitation. The second factor is the "extrinsic family influence" including reward, family coercion and sponsorship.

In order to test whether the two-factor solution was appropriate and parsimonious in describing the latent factors, two additional factor solutions (one-factor and three-factor) were evaluated. For the one-factor solution, the model explained $46.4 \%$ of variance. Yet the eigenvalue of the next possible factor was higher than unity, implying the emergence of a second factor. For the three-factor solution, the model explained $71.22 \%$ of variance. Although no double loading occurred using the criterion of factor loading greater than .57 , the eigenvalue of the third factor was only .65 , much lower than unity. This implies the third factor is not stable. Thus the two-factor solution was selected because all eigenvalues were greater than unity and the factor structure was the most distinguished and the clearest.

To ensure the stability of the factor structure, factor analyses for two sub-samples (subsample A: case 1 to case 2973; subsample B: case 2974 to case 5946) were performed. In principal component analyses with varimax rotation, two factors emerged. The eigenvalues of all factors were greater than unity. For subsample A, the two-factor model explained $63.50 \%$ of variance whereas the two-factor model for sub-sample B explained $64.47 \%$. The loading was identical to the overall sample. The coefficients of congruence in these two sub-samples were .99 (factor 1) and .99 (factor 2), which are very high. In short, the factor structure consisted of two stable factors. The first factor was named "positive family influence" (PFI) whereas the second factor was named "extrinsic family influence" (EFI). These two factors were positively correlated between themselves (Pearson's $r=.43$ ).

\section{Reliability of C-FIAV and its Sub-Scales}

The overall internal consistency of the 9-item C-FIAV was very good (Cronbach's $\alpha=.85$ ). The alpha if one item was deleted ranged from .82 to .85 . The mean inter-item correlation was .37 . The mean item-total was .57. The corrected item-total correlation ranged from .31 to .71 , which indicated good correlation. The Cronbach's $\alpha$ for the two subscales were .86 (PFI) and .75 (EFI). The scale showed good internal consistency.

\section{Criterion-Related Validity of C-FIAV Subscales}

It was hypothesized that volunteers' positive family influence would be higher than non-volunteers and volunteers' 
extrinsic family influence would be lower than nonvolunteers. Univariate analysis was performed to detect whether there was difference between volunteers and nonvolunteers regarding the two sub-scales of C-FIAV. The mean PFI score for non-volunteers within the past 12 months was $3.47(\mathrm{SD}=1.02)$ whereas the mean score for volunteers was 3.68 (SD = 1.02). The PFI score for volunteers was higher than non-volunteers $(t(5930)=-7.87, p<.001)$. The effect size, Cohen's d, was .43 , which indicated a medium difference. The mean EFI score for non-volunteers was 2.73 $(\mathrm{SD}=1.12)$ whereas the mean score for volunteers was 2.54 $(\mathrm{SD}=1.09)$. The EFI score for non-volunteers was higher than volunteers $(t(5929)=6.60, p<.001)$. The effect size, Cohen's $d$, was .17 , which indicated a small difference. The overall C-FIAV score for non-volunteers was 3.22 (SD = .91) whereas the mean score for volunteers was 3.30 (SD $=$ 90). The overall C-FIAV score for volunteers was higher than non-volunteers $(t(5930)=-3.21, p<.001)$. The effect size, Cohen's $d$, was .08, which indicated a small difference. The C-FIAV can differentiate between non-volunteers and volunteers. It showed good criterion-related validity.

\section{Construct Validity of C-FIAV}

In addition to C-FIAV, respondents were required to rank the importance of family, school and peers to their (1) decision to volunteer and their (2) continuation to volunteer. The ranking scores of these two questions were added respectively as composite ranks for family, school, and peers. The lower the numerical composite rank, the greater the importance of that system the respondent places. On the other hand, the higher the C-FIAV score, the greater the influence of that system. Each of the composite rank for family, school and peers were correlated with the C-FIAV. Construct validity was obtained when the C-FIAV scores and the family rank were negatively associated.

The result showed that negative and significant relationship was only found for family rank and C-FIAV (rho $=-.26$, $p<.001)$. C-FIAV held positive associations with school rank $($ rho $=.08, p<.001)$ and peer rank $($ rho $=.12, p<.001)$. For the subscales, PFI held negative and significant relationship with family rank (rho $=-.26, p<.001)$, but held positive associations with school rank (rho $=.10, p<.001)$ and peer rank (rho $=.14, p<.001)$. EFI held negative and significant relationships with family rank (rho $=-.16, p<.001$ ), but did not hold significant relationships with school rank and peer rank. It means that for those who marked higher family influence in C-FIAV, they also ranked the influence of family higher. C-FIAV showed good construct validity.

\section{Normative Findings for Family Influences}

In general, adolescents perceived family influence as positive (Table 2). Several observations were highlighted from the findings: less than half of respondents (49\%) reported that family members volunteered such as serving company volunteer service groups and churches; around seventy-seven per cent regarded families considered volunteering as meaningful; more than seventy per cent of adolescents found their families supportive.

Table 2. Item Statistics for Familial Influence Scale with Gender Difference and Grade Difference

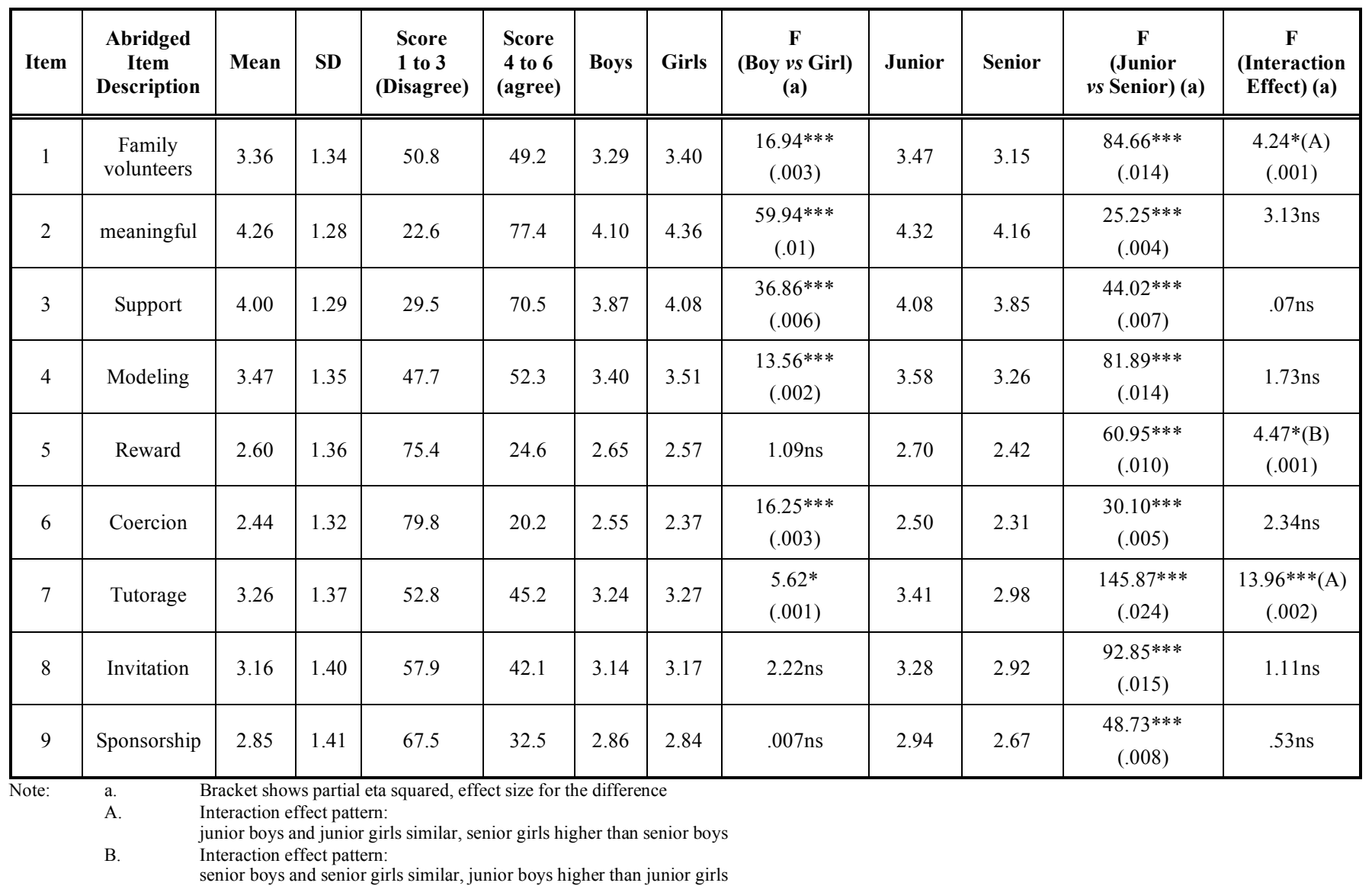




\section{Gender and Grade Effects on the C-FIAV and its Sub- scales}

Three two-way analyses of variance were performed to examine the effects of gender and grade on the C-FIAV and its subscales. For C-FIAV, girls' family influence was higher than boys with very small effect size $(\mathrm{F}(1,5928)=10.87, p$ $<.01$, partial $\left.\eta^{2}=.002\right)$. Juniors' family influence was higher than seniors with moderate effect size $(F(1,5928)=144.05$, $p<.001$, partial $\left.\eta^{2}=.024\right)$. Interaction effect was also significant with very small effect size $(\mathrm{F}(1,5928)=5.99, p<$ .05 , partial $\left.\eta^{2}=.001\right)$. Post-hoc LSD analysis showed that while the family influences for junior boys and junior girls were similar, the family influence for senior girls was higher than senior boys.

Girls' PFI was higher than boys with small effect size (F $(1,5928)=30.14, p<.001$, partial $\left.\eta^{2}=.005\right)$. Juniors' PFI was higher than seniors with moderate effect size ((F (1, $5928)=129.61, p<.001$, partial $\left.\eta^{2}=.021\right)$. The grade effect was stronger than gender effect on PFI. Interaction effect was also significant with very small effect size $(\mathrm{F}(1,5928)=$ $5.18, p<.05$, partial $\left.\eta^{2}=.001\right)$. Post-hoc LSD analysis showed that while the PFI for junior boys and junior girls were similar, the PFI for senior girls was higher than senior boys.

Girls' EFI was lower than boys with small effect size ( $\mathrm{F}$ $(1,5927)=5.04, p<.05$, partial $\left.\eta^{2}=.001\right)$. Juniors' EFI was higher than seniors with moderate effect size $((\mathrm{F})(1,5927)=$ 69.76, $p<.001$, partial $\left.\eta^{2}=.012\right)$. The grade effect was stronger than gender effect on EFI. The interaction effect was not significant.

\section{DISCUSSION}

The study aims to develop and validate a scale to measure family influence to adolescent volunteerism as there is no such scale in existing literature. The expert panel generally agreed that the C-FIAV had good content validity. Conceptually speaking, there are two domains of family influence. Empirical findings also yielded these two factors. They are called the positive family influence and the extrinsic family influence.

The C-FIAV attained good internal consistency. Criterion-related validity was also attained as the scale could differentiate volunteers and non-volunteers. In general, adolescents are close to families [2]. If the family influence is more positive on volunteerism, there is a higher tendency for adolescents to participate in volunteer service. Volunteers rated the positive family influence higher than non-volunteers, implying that they have recognized their family members' participation and positive beliefs on volunteerism. They were also affected more by positive family support, family modeling, tutorage and invitation. On the other hand, nonvolunteers rated higher on coercion, reward and sponsorship. These three kinds of family influence might deter nonvolunteers to participate in volunteer service, as they might only view services as opportunities to obtain reward and sponsorship. Construct validity was attained as the family influence score was directly related to adolescents' perception of the ranking importance of family with other social systems such as schools and peers.
Existing studies on family influence tend to focus on family support and modeling, both of which belong to positive family influence. Yet extrinsic influence was not even mentioned in volunteering literature. The theory of extrinsic and intrinsic motivation [17] can offer the explanation for the existence of extrinsic influence. Both types of family influence should be examined.

Extrinsic family influence includes tangible reward and monetary sponsorship. Intrinsic motivation is derived from the satisfaction of serving with a sense of control and competence [23]. Intrinsic motivation depends on the service nature and process, whereas extrinsic influence normally depends on task completion, i.e. you obtain the reward once you perform. Extrinsic rewards are not conducive to intrinsic motivation, especially when rewards are expected, tangible and performance-contingent [23]. Extrinsic rewards take adolescents' attention away from intrinsic ones and they may never appreciate the inherent rewards that a task will provide [24].

One item of extrinsic influence was related to coercion to volunteer. Family members may hope that adolescents can join volunteer service to the extent that adolescents feel the pressure. This results in coercion. Coercion is a kind of heteronomy, i.e. controlled regulation [23]. Overt pressure deters adolescents from further volunteer service. Since adolescents feel they lack autonomy, they form a negative impression towards volunteering.

One may argue that the extrinsic influence should be separated into the reinforcement and coercion domains. However, the principal components analysis showed that for the three-factor solution, these two domains could not be delineated. In fact, the factor loadings for reward and coercion were strikingly similar. The empirical evidence rejected further differentiation of the extrinsic influence.

It should be noted that extrinsic family influence is not equal to negative family influence. In fact, it held medium positive association with positive family influence. Extrinsic family influence does not cancel the effect of positive family influence. Both domains of family influence work together to influence adolescent volunteering behavior. Further studies should be focused on substantiate the relationship between extrinsic and positive family influences. The relationship may be more complicated than it seems to be, involving either non-linear associations or linear relationships with asymptote (turning point) or plateau (saturation).

In addition to the construction of the C-FIAV and validation, this study also explores some descriptive findings of the scale from the vast sample. The top three kinds of family influence recognized by adolescents were family belief of volunteering as meaningful, family support and family modeling. Around $70 \%$ of adolescents found the families supportive, whereas only $24 \%$ of Hong Kong adolescents found the families supportive twenty years ago [11]. The atmosphere of volunteerism among youths was more proactive after the handover of Hong Kong to China in 1997. Policy addresses had emphasized volunteerism as a cornerstone of youth development and social capital [25]. Both government and non-governmental organizations organized many services for adolescents. It was speculated that under the environment conducive to the development of adolescent volunteerism more parents accepted and supported adolescent children's 
volunteer service. Parents did not push the adolescents to volunteer, as reflected from the low average score of family coercion. In general, adolescents also perceived families would not use reward or sponsorship to induce them to volunteer as well.

Although item analysis yielded some interesting results toward specific family influence, the profile tended not to be stable and reliable. Each kind of influence was represented by one item. The strength of individual item as a representation of an area was weaker than grouped items.

When the domains of family influence were compared, boys were more aware of the extrinsic family influence whereas girls were more aware of the positive family influence. There are two explanations. First, girls in literature are more prosocial than boys [26]. Girls are more empathic and prosocial than boys [27]. Female role tends to focus more on caring and sympathy to others. It also places a higher value to helping others. Male role however tends to focus on personal competence and success. Male role is not perceived to be caring. Role theory suggests that girls are more sensitive to the prosocial and caring atmosphere of the families. Therefore they rate the corresponding family influence higher than boys. However, boys tend to view volunteer service as an opportunity to obtain reward or to give in to a power struggle (coercion). This fits the male gender role [21]. They are more alert to tangible reward and competence or power and are more sensitive to the extrinsic family influence to volunteerism.

The grade effect was in fact more pronounced than the gender effect. Family influence for juniors was rated higher than seniors. Developmental perspective can be used to explain the observation [28]. Junior adolescents are influenced by families more than other social systems. However, senior adolescents are more influenced by other systems that the relationship with the families was less close. Such systems include schools, peers, or even romantic relationship [29]. Subsequently, juniors rated family influence higher than seniors.

There are several implications of the study for family research and practice. First, the construction of C-FIAV is an initial attempt in existing literature to assess the overall family influence in a systematic manner with a conceptual framework. The list of influence may not be exhaustive. Not all assumingly positive family influence can exert empirically positive effect, especially reward and sponsorship. The findings enable us to understand more about Chinese family influence on adolescent volunteerism. This contribution is important because there are few available measures of psychosocial functioning in the Chinese culture [30].

The second implication of the findings is that the development of the C-FIAV and the accumulation of research findings can enable volunteer service organizers to assess different aspects of family influence in Chinese culture in an objective manner. Practitioners can assess the family influence of the adolescents. Family members can be educated about the importance of various forms of influence. The effectiveness of this training program can be assessed by the use of the scale. This move is important as the HKSAR government tried to advocate family members to motivate their children to volunteer since 2007 [31].
The third implication of the findings is that gender and age were related to the C-FIAV subscale scores. This suggests that it is important to be sensitive to gender and age differences in the level of family influence to volunteerism. It seems that senior boys were the least affected by family influence on volunteerism. The transition of family influence from junior grades to senior grades for boys can be further explored. Subsequent intervention plans should be formulated to enhance the influence. In addition, different norms should be developed for male and female Chinese adolescent volunteers with different grades. In fact, with the number of participants of this validation, norms can be generated so that we can see the trend of family influence for four groups of adolescents (junior boys, senior boys, junior girls, senior girls).

Finally, the current study proposes the possibility of the construction of identical scales with other significant social systems for adolescents such as schools and peers. The influence of various systems can be compared and the significance of family influence can be explicated.

There are several limitations of the current study. First, because the assessment of family influence was based on self-report measures from the perspective of the adolescents only, the use of multiple perspectives would constitute a better strategy to assess the constructs under study. In particular, the employment of responses based on parents would be helpful. Second, the research findings reported in the current study are based on adolescents in Hong Kong. There is a need to replicate the findings in other Chinese contexts. In addition, though the sample size was large, they were not randomly sampled. The generalizability of the findings to other Chinese adolescent populations should be interpreted with caution. Third, the current findings provided some good evidence for factorial validity, criterion-related validity and construct validity. However, more central evidence on other aspects of the validity of the measure, including convergent and discriminant validities, were not explored in this study [32]. Fourth, we can add negative family influence so that a wider scope of influence can be detected. We should not under-estimate the pull factor from families, especially many Chinese families focus on adolescents' studying as the sole activity of paramount importance [33]. Other activities including volunteering participation should not affect studying. Finally, the current study has adopted the conceptual model from the motivational cognitive approach to understand family influence. Confirmatory factor analysis can further be employed to explore whether the solution has the best model fit.

\section{ACKNOWLEDGEMENT}

The preparation for this paper was financially supported by Wofoo Foundation.

\section{REFERENCES}

[1] Hartup WW. The company they keep: Friendships and their developmental significance. Child Dev 1996; 67: 1-13.

[2] Offer KN, Church RB. Generation gap. In: Lerner RM, Petersen AC, Brooks-Gunn J, Eds. Encyclopedia of adolescence. New York: Garland 1991.

[3] Hofer M. Community service and social cognitive development in German adolescents. In: Yates M, Youniss J, Eds. Roots of civic identity: International perspectives on community service and activism in youth. Cambridge: Cambridge University Press 1999. 
[4] Omoto AM, Snyder M. Considerations of community: The context and process of volunteerism. Am Behav Sci 2002; 45(5): 846-67.

[5] Pancer SM, Pratt MW. Social and family determinants of community service involvement in Canadian youth. In: Yates M, Youniss J, Eds. Roots of civic identity: International perspectives on community service and activism in youth. Cambridge: Cambridge University Press 1999.

[6] Yates M, Youniss J. A developmental perspective on community service in adolescence. Soc Dev 1999; 5(1): 85-111.

[7] Clary EG, Miller J. Socialization and situational influences on sustained altruism. Child Dev 1986; 57: 1358-69.

[8] Engaging youth in lifelong service: Findings and recommendations for encouraging a tradition of voluntary action among America's youth [homepage on the internet]. United States: Independent sector; c2008 [updated 2008 Dec 8, cited 2008 Dec 9, from http://www.independentsector.org/programs/research/engagingyout h.html.

[9] Fletcher AC, Elder GHJr, Mekos D. Parental influences on adolescent involvement in community activities. J Res Adolescence 2000; 10(1): 29-48.

[10] Agency for Volunteer Service. A report on public perception towards volunteering: A follow-up study. Hong Kong: Author 1994.

[11] Central Committee on Youth. Report on youth policy. Hong Kong: Author 1988.

[12] Law BMF. Volunteer service participation among secondary school students in Hong Kong. PhD [dissertation]. Hong Kong: Chinese University of Hong Kong; 2008.

[13] Lu L. Understanding happiness: A look into the Chinese folk psychology. J Happiness Stud 2001; 2: 407-32.

[14] Bond MH, Hwang KK. The social psychology of Chinese people. In: Bond MH, Ed. The psychology of the Chinese people. Oxford: Oxford University Press 1987.

[15] Cialdini RB, Kallgren CA, Reno RR. A focus theory of normative conduct: A theoretical refinement and reevaluation of the role of norms in human behavior. In: Berkowitz L, Ed. Advances in experimental social psychology (Vol. 24). New York: Academic Press 1991.

[16] Hodgkinson VA. Key factors influencing care, involvement, and community. In: Schervish PG, Hodgkinson VA, Gates M, Eds. Care and community in modern society: Passing on the tradition of service to future generations. San Francisco: Jossey-Bass 1995.

[17] Deci EL, Ryan RM. Intrinsic motivation and self-determination in human behavior. In: Steers RM, Porter LW, Eds. Motivation and Work Behavior. New York: McGraw-Hill 1991.
[19] Bronfenbrenner U. Environments in developmental perspective: Theoretical and operational models. In: Friedman SL, Wachs TD, Eds. Measuring environment across the life span: Emerging methods and concepts. Washington, DC: American Psychological Association 1999.

[20] Tolan PH, Gorman-Smith D, Henry DB. The developmental ecology of urban males' youth violence. Dev Psychol 2003; 39 (2):27491 .

[21] Ruble TL. Sex stereotypes: Issues of change in the 70s. Sex Roles 1983; 9: $397-402$.

[22] Shek DTL. Parenting characteristics and adolescent psychological well-being: A longitudinal study in a Chinese context. Genet Soc Gen Psychol Monogr 1999; 125(1):27-44.

[23] Ryan RM, Deci EL. Self-regulation and the problem of human autonomy: Does psychology need choice, self-determination and will? J Pers 2006; 7 (6): 1557-85.

[24] Greene D, Sternberg B, Lepper MR. Overjustification in a token economy. J Pers Soc Psychol 1976; 34: 1219-34.

[25] Hong Kong Government. Policy address. Hong Kong: Government Printer 1997.

[26] Flanagan CA, Bowes JM, Honsson B, Csapo B, Sheblanora E. Ties that bind: Correlates of adolescents' civic commitments in seven countries. J Soc Issues 1998; 54(3):457-75.

[27] Eisenberg N, Shea CL, Carlo G, Knight GP. Empathy-related responding and cognition: A "chicken and the egg" dilemma. In: Gewirtz WM, Kurtines JL, Eds. Handbook of moral behavior and development (Vol.2). Mahwah, NJ: Lawrence Erlbaum 1991.

[28] Steinberg L, Morris AS. Adolescent development. Annu Rev Psychol 2001; 52: 83-110.

[29] Bouchey HA, Furman W. Dating and romantic experiences in adolescence. In: Adams GR, Berzonsky MD, Eds. Blackwell handbook of adolescence. London: Blackwell 2003.

[30] Shek DTL, Ed. Research on social work practice in Chinese communities. Res Soc Work Pract 2002; 12: 485-581.

[31] Central Office for Volunteer Service [homepage on the Internet]. Hong Kong: Social Welfare Department; c2005 [updated 2008 July 31; cited 2008 August 30]. Available from: http://www.swd.gov.hk/en/index/site_pubsvc/page_supportser/sub_ centraloff/.

[32] Springer DW, Abell N, Hudson WW. Creating and validating rapid assessment instruments for practice and research: Part 1. Res Soc Work Pract 2002; 12 (3): 408-39.

[33] Commission on Youth. Study on civic awareness and moral values of youth. Hong Kong: Author 1998. 